\title{
Protease-activated receptor 1 inhibitor improves brain edema in rats with intracerebral hemorrhage
}

\author{
Huang $\mathrm{P}^{1}, \mathrm{He} \mathrm{XY}^{2}, \mathrm{Xu} \mathrm{M}^{3}$ \\ Department of Neurology, The second People's Hospital of Deyang City, Minjiang West Road, DeYang, \\ Sichuan, China.782067723@qq.com
}

\begin{abstract}
AIM: To investigate the changes of water content in brain tissue, the expression of AQP4mRNA after cerebral hemorrhage in rats, and the intervention effect of Protease activated receptor 1 inhibitor (PAR1 inhibitor) on both. METHODS: Establish sham operation group (Sham group), ICH group, ICH+PAR1 inhibitor high-dose group (PI(H)group), ICH+PAR1 inhibitor low-dose group (PI(L)group), 25 in each group. Neural dysfunction scores were performed at $1 \mathrm{~d}, 3 \mathrm{~d}, 7 \mathrm{~d}, 14 \mathrm{~d}$, and $21 \mathrm{~d}$ after surgery, and brain water content and AQP4mRNA content were measured.

RESULTS: Results: The neurological dysfunction and cerebral edema of rats with cerebral hemorrhage reached the peak at 3 days after operation. With the increase of time, the water content and AQP4mRNA content in the $\mathrm{PL}(\mathrm{H})$ group were higher than those in the $\mathrm{PI}(\mathrm{L})$ group. The differences were statistically significant.

CONCLUSIONS: Appropriate inhibition of PAR1 can alleviate cerebral edema around the hematoma and play a role in improving the function of nerve defects. The mechanism may be realized by down-regulating the expression of AQP4mRNA in brain tissue (Tab. 3, Fig. 3, Ref. 25). Text in PDF www.elis.sk KEY WORDS: cerebral hemorrhage, protease activated receptor 1 (PAR1), AQP4mRNA.
\end{abstract}

\section{Introduction}

In recent years, the incidence of cerebral hemorrhage ( $\mathrm{ICH})$ has increased year by year. Due to the non-renewable nature of cerebral neurons, patients with cerebral hemorrhage often have severe neurological dysfunction, which places a heavy burden on society and families (1). Cerebral edema is an inevitable pathophysiological process after ICH. It can increase intracranial pressure and aggravate secondary damage to neurons in the brain. It is an important cause of clinical deterioration of patients with ICH (2). Therefore, actively exploring ways to reduce cerebral edema after ICH is of great significance for reducing the mortality of the disease and improving the prognosis (3).

Aquaporin 4 (AQP4) is a special membrane protein, which is located between $18 \mathrm{q} 11.2$ and q12.1. Its main role is to regulate the water balance in the body (4). Studies show that AQP4 is also widely expressed in the central nervous system, especially overexpressed on astrocytes (5). Thrombin is a highly specific serine proteolytic enzyme. After cerebral hemorrhage, a large amount of thrombin is produced and activated for various reasons. Recently,

${ }^{1}$ Department of Neurology, People's Hospital of Deyang City, DeYang, Sichuan, China, ${ }^{2}$ Department of Neurology, The Affiliated Hospital of Southwest Medical University, Jiangyang District, Cangzhou City, Sichuan Province, LuZhou, SiChuan, China, and ${ }^{3}$ Department of Neurology, The second People's Hospital of Deyang City, DeYang, Sichuan, China.

Address for correspondence: Min Xu, Department of Neurology, The second People's Hospital of Deyang City, 340 Minjiang West Road, DeYang, Sichuan, 618000, China.

Phone: +8615228287552, Fax: 08386621111 studies have shown that thrombin can bind to thrombin receptor 1 (PAR1) and then down-regulate the expression of AQP4mRNA in the brain tissue of experimental ICH rats to reduce brain edema after ICH (6). Further research found that thrombin is a doubleedged sword for $\mathrm{ICH}$, and its specific effect depends on the extent to which the thrombin receptor (PAR1) is activated, that is, high concentrations of thrombin can damage brain tissue, while low concentrations of coagulation enzymes have protective effects on brain tissue $(7,8)$. Then, we can ask the following questions: Do different degrees of PAR1 activation have different effects on cerebral edema after ICH? Based on this, this study uses an experimental rat cerebral hemorrhage model, using PAR1 as the entry point, to explore the effects of different degrees of PAR1 activation on brain water content and AQP4mRNA expression after cerebral hemorrhage and possible molecular mechanisms. Rehabilitation offers new ideas.

\section{Materials and methods}

Animals

100 healthy adult male SD rats, aged 6-9 weeks, average (8.3 \pm 0.6$)$ weeks, weighing approximately $260-310 \mathrm{~g} / \mathrm{head}$, with average $283.41 \pm 12.23 \mathrm{~g}$.

\section{Animal grouping and administration}

Before the experiment, $100 \mathrm{SD}$ rats were immediately divided into Sham group, $\mathrm{ICH}$ group, $\mathrm{ICH}+\mathrm{PI}(\mathrm{H})$ group, $\mathrm{ICH}+\mathrm{PI}(\mathrm{L})$ group, 25 rats in each group, and rats in each group were randomly divided into $1 \mathrm{~d}$ and $3 \mathrm{~d}, 7 \mathrm{~d}, 14 \mathrm{~d}, 21 \mathrm{~d}$, five rats at each time point. Cerebral 
hemorrhage model was established by using references. The Sham group only switched the holes without injecting blood (24). After 2 hours of successful modeling, the Sham group and the ICH group were intraperitoneally injected with $2 \mathrm{ml}$ of normal saline, while the $\mathrm{PI}(\mathrm{H})$ group was administered with SCH79797 $250 \mathrm{ug} / \mathrm{kg}$, and the PI(L) group was administered with SCH79797 $25 \mathrm{ug} / \mathrm{kg}$. Intraperitoneal injection was performed in $2 \mathrm{ml}$ of physiological saline.

\section{Neurological dysfunction score}

After the rats were awake, the four-point scoring method from the literature was used to evaluate the model of the experimental rats, and those who failed in the modeling were eliminated, and the rats were randomly supplemented (9). The neurological scores of experimental rats were scored using references. The higher the score, the more severe the neurological impairment (10).

\section{Material}

The model rats were anesthetized with $1 \%$ sodium pentobarbital at $1 \mathrm{~d}, 3 \mathrm{~d}, 7 \mathrm{~d}, 14 \mathrm{~d}$, and $21 \mathrm{~d}$ at the corresponding time points. After aortic perfusion with $4 \%$ paraformaldehyde and internal fixation, the rats were decapitated and killed quickly. Brain tissue samples were collected for: 1) determination of changes in the water content of the brain tissue around the hematoma (dry and wet weight method); 2) reverse transcription PCR to detect the expression of AQP4mRNA around the hematoma.

\section{Determination of water content in brain tissue}

Quickly cut the tissue with brain water content into $2 \mathrm{~mm}$ thick pieces, place it in a weighing bottle, weigh the wet weight with an electronic balance (accuracy: $0.1 \mathrm{mg}$ ), and place it in a $105^{\circ} \mathrm{C}$ constant temperature oven. Dry to constant weight. Take the difference between the last two dry weights as less than 0.2 $\mathrm{mg}$ as the constant weight standard. Put the brain tissue into the same weighing bottle again to weigh the dry weight. Calculate the brain tissue water content (\%) according to Elliott's formula $=($ wet weight-dry weight $) /$ wet weight $\times 100 \%$.

\section{Total RNA extraction}

Weigh $100 \mathrm{mg}$ of brain tissue surrounding the hematoma to extract total RNA according to the instructions of the TRIzol kit. The RNA standards with reliable quality are those whose purity and concentration are between 1.8-2.1 and above 1.5ug/ul, respectively.

\section{cDNA Synthesis and PCR}

The M-MLV reverse transcriptase kit (Invitrogen TM) was used to prepare the reaction system. Prepare the reverse transcription PCR reaction mixture in a 96-well reaction plate. The AQP4mRNA primer sequence is: For-ward: 5'-GAGACGAGAAGAAGGGGAAGG-3 ', Reverse: 5'-AATCTGAGGCCAGTTCTAGGGA-3'. Since the Ct value in PCR cannot be analyzed in statistics as raw data, it needs to be standardized. In this study,

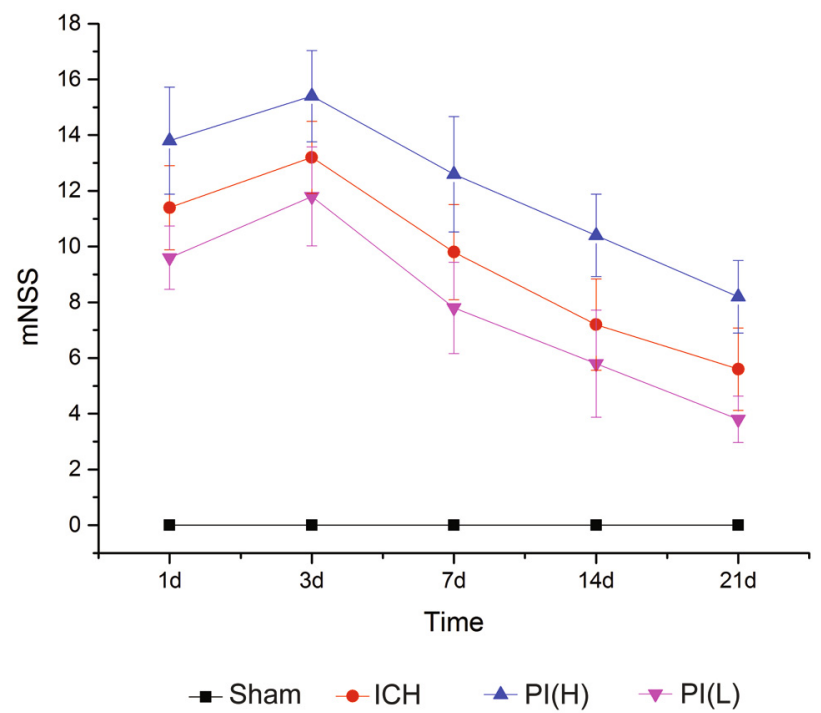

Fig. 1. Nerve function scores of experimental rats at various time points.

$2^{-\Delta \mathrm{Ct}}$ is used to represent the relative expression of microRNA, and $\Delta \mathrm{Ct}=($ target microRNACt value- $\mathrm{U} 6 \mathrm{Ct}$ value $)$.

\section{Statistical analysis}

The experimental data were analyzed by SPSS 17.0 software. The obtained data were expressed as mean \pm standard deviation $(\mathrm{x} \pm \mathrm{s}$ ), with both sides $\alpha=0.05$ as the test standard, and $\mathrm{p}<0.05$ was considered statistically significant. The homogeneity of variance was determined by one-way ANOVA (one-way ANOVA) LSD method.

\section{Results}

Neural function scores at various time points in experimental rats

The results show that high doses of PAR1 inhibitors are not conducive to neural function recovery, while low doses of PAR1 inhibitors can promote neural function recovery (Tab. 1 and Fig. 1).

\section{Changes in brain tissue water content}

With time, $\mathrm{PI}(\mathrm{H})$ brain tissue water content is higher than in the ICH group, while PI(L) group brain water content is lower than in the ICH group, suggesting that high dose of PAR1 inhibitor will aggravate cerebral edema after cerebral hemorrhage, and low dose of PAR 1 inhibitors reduce brain edema after intracerebral hemorrhage (Tab. 2 and Fig. 2). 
Tab. 2. Brain tissue water content changes at various time points in experimental rats $(x \pm \mathrm{s})$.

\begin{tabular}{lcccccc}
\hline Item & $\mathrm{n}$ & $1 \mathrm{~d}$ & $3 \mathrm{~d}$ & $7 \mathrm{~d}$ & $14 \mathrm{~d}$ & $21 \mathrm{~d}$ \\
\hline Sham & 5 & $69.60 \pm 1.14$ & $70.80 \pm 1.30$ & $70.40 \pm 2.07$ & $67.98 \pm 1.78$ & $67.48 \pm 1.52$ \\
ICH & 5 & $83.80 \pm 1.30^{*}$ & $86.80 \pm 1.78^{*}$ & $81.60 \pm 1.51 *$ & $76.71 \pm 1.54^{*}$ & $71.54 \pm 1.48^{*}$ \\
PI(H) & 5 & $86.00 \pm 1.58^{*} \#$ & $89.00 \pm 1.58^{*} \#$ & $83.89 \pm 1.92^{* \#}$ & $79.41 \pm 1.67 * \#$ & $75.78 \pm 1.52 * \#$ \\
PI(L) & 5 & $81.60 \pm 1.14^{* a}$ & $84.00 \pm 1.58^{* a}$ & $79.40 \pm 2.07 * a$ & $72.35 \pm 2.12^{*} \mathrm{a}$ & $68.27 \pm 1.65 * \mathrm{a}$ \\
\hline
\end{tabular}

Compared with the Sham group, ${ }^{*} \mathrm{p}<0.05$; compared with the ICH group, $\# \mathrm{p}<0.05$; compared with the ICH group, a $\mathrm{p}<0.05$

Tab. 3. Relative expression of AQP4mRNA in brain tissue at various time points in experimental rats after operation $(x \pm s)$.

\begin{tabular}{lcccccc}
\hline Item & $\mathrm{n}$ & $1 \mathrm{~d}$ & $3 \mathrm{~d}$ & $7 \mathrm{~d}$ & $14 \mathrm{~d}$ & $21 \mathrm{~d}$ \\
\hline Sham & 5 & $0.78 \pm 0.05$ & $0.86 \pm 0.04$ & $0.79 \pm 0.03$ & $0.78 \pm 0.04$ & $0.77 \pm 0.05$ \\
ICH & 5 & $1.02 \pm 0.10^{*}$ & $1.20 \pm 0.11^{*}$ & $1.12 \pm 0.09^{*}$ & $1.08 \pm 0.11^{*}$ & $0.91 \pm 0.08^{*}$ \\
$\mathrm{PI}(\mathrm{H})$ & 5 & $1.21 \pm 0.12^{* \#}$ & $1.45 \pm 0.14^{*} \#$ & $1.31 \pm 0.11^{*} \#$ & $1.24 \pm 0.12^{*} \#$ & $1.14 \pm 0.09^{*}$ \\
PI(L) & 5 & $0.82 \pm 0.07 * \mathrm{a}$ & $1.02 \pm 0.10^{*} \mathrm{a}$ & $0.97 \pm 0.09^{* a}$ & $0.91 \pm 0.08^{* a}$ & $0.78 \pm 0.08^{* a}$ \\
\hline
\end{tabular}

Compared with the Sham group, ${ }^{*} \mathrm{p}<0.05$; compared with the ICH group, $\# \mathrm{p}<0.05$; compared with the ICH group, a $\mathrm{p}<0.05$

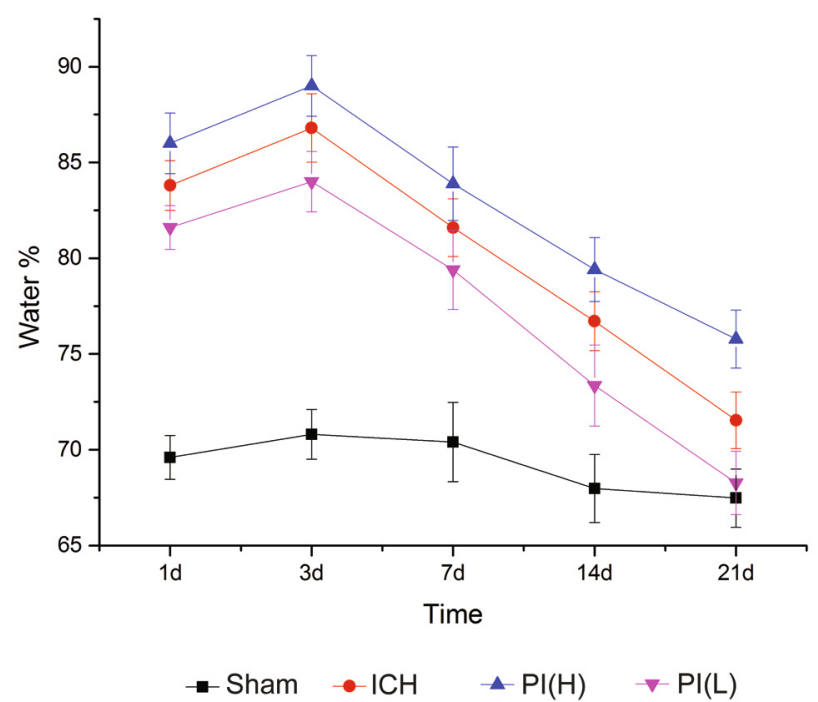

Fig. 2. Brain tissue water content changes at various time points in experimental rats.

\section{Relative expression of $A Q P 4 m R N A$ in the brain group}

Over time, AQP4mRNA in $\mathrm{PI}(\mathrm{H})$ brain tissue was higher than that in ICH group, while AQP4mRNA in brain tissue of PI(L) group was lower than that in ICH group, indicating that high-dose PAR1 inhibitors will promote AQP4mRNA expression, while lowdose PAR1 inhibitor AQP4mRNA expression (Tab. 3 and Fig. 3).

\section{Discussion}

Cerebral edema refers to the phenomenon that brain volume increases and intracranial pressure increases due to abnormal accumulation of water in brain tissue. Due to the continuous increase of intracranial pressure, once it exceeds the compensation range of normal intracranial pressure, it can cause changes in the structure or function of the brain group or even the formation of cerebral hernia, which seriously affects the prognosis of patients with brain injury.

Cerebral edema caused by ICH mainly includes vascular edema caused by bloodbrain barrier destruction and cell-derived edema caused by tissue ischemia and hypoxia. Although different types of brain edema have different causes, it has been clear that all brain edemas are with the involvement of aquaporin 4 (AQP4) $(12,13)$. AQP4 is widely distributed in the central nervous system. Its molecular structure is a channel-like tetramer structure. This channel has a structure that blocks ions (14). Each of these monomers forms an independent water channel. Therefore, the four monomers have independent Of activity. Multiple studies have found that AQP4 plays an important role in the formation and elimination of cerebral edema (15). The results of this study show that in the ICH group, $\mathrm{PI}(\mathrm{H})$ group, and $\mathrm{PI}(\mathrm{L})$ group, the water content of the brain tissue surrounding the hematoma has begun to increase on the 1st postoperative day, and reaches a peak on the 3 rd postoperative day, and then over time. The water content in the brain group gradually decreased until 14 days after the operation, which was still higher than that in the Sham group; and the trend of the relative expression of AQP4mRNA in the brain tissue around the postoperative hematoma was basically consistent with the change in the water content in the brain group. The brain tissue water content and relative expression of AQP4mRNA at each time point were higher than those of the Sham group $(P<0.05)$, which confirmed that AQP4 was involved in the occurrence of cerebral edema after ICH. This is similar to the conclusions of others (16).

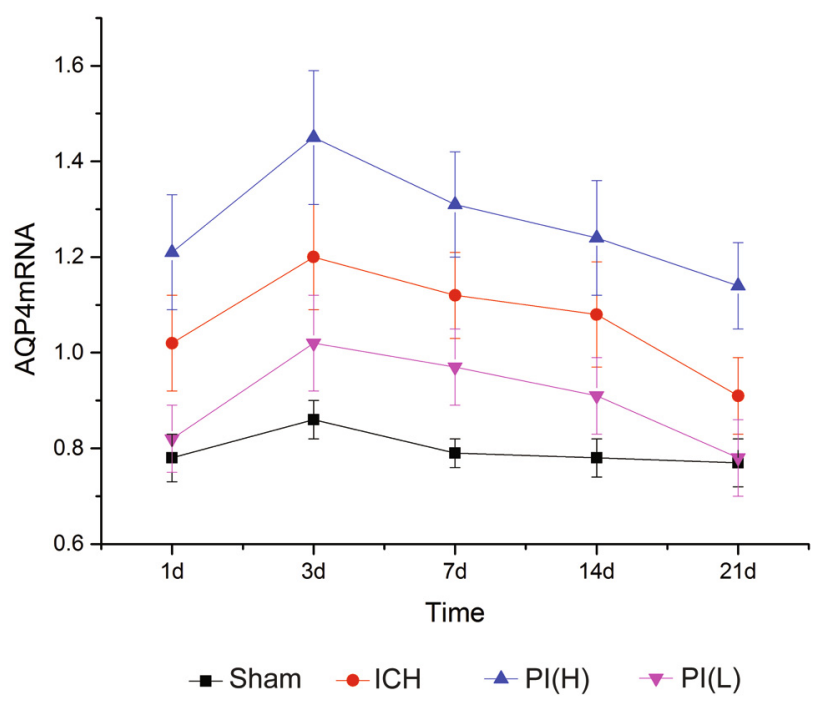

Fig. 3. Relative expression of AQP4mRNA in brain tissue at various time points in experimental rats after operation. 
After ICH, due to the cascade of hematomas, a large number of thrombin synthesis scores can be activated. Studies show that thrombin activation in ICH is not only related to the body's prevention of hematoma expansion (17), but also to the formation of cerebral edema (18). The mechanism is that the combination of thrombin and PAR1 causes PAR1 to be activated, causing phospholipase C (PLC) to be activated on the cell membrane. Activation and decomposition into phosphatidylinositol diphosphate 2(PIP2) produces diglyceryl glycerol (DG) and inositol triphosphate 3(IP3). Promote intracellular $\mathrm{Ca}^{2+}$ overload through DG and IP3 signaling pathways (19).Thus, the activation of multiple signaling pathways in the cell eventually leads to the upregulation of AQP4mRNA expression. Studies have found that the use of a thrombin-specific inhibitor hirudin can effectively inhibit the occurrence of cerebral edema after ICH (20), and the mechanism may be achieved by down-regulating AQP4mRNA expression and further down-regulating AQP4 (21), Thrombin can play various roles only in combination with PAR1, and studies have found that the peak expression of PAR 1 after ICH in rats is consistent with the peak of cerebral edema and baicalin can reduce cerebral edema symptoms by inhibiting PAR-1 expression in brain tissue after ICH (22), suggesting that PAR1 is indeed involved in the occurrence and development of cerebral edema after cerebral hemorrhage (23), therefore, theoretically, by appropriately inhibiting PAR1, blocking the abovementioned cellular signal transduction pathway can reduce brain tissue edema. However, some studies have shown that cerebral edema and neurological dysfunction after cerebral infarction in PAR1 knockout rats are worse than those in the control group, suggesting that severe PAR1 deficiency after brain injury is not conducive to reducing brain tissue edema (24). In this study, we observed that in the $\mathrm{PI}(\mathrm{H})$ group compared with $\mathrm{ICH}$ group, the relative expression of AQP4mRNA increased and brain water content increased at various time points after surgery $(p<0.05)$, suggesting that excessive PAR1 inhibitors may be upregulated. The expression level of AQP4mRNA further aggravates cerebral edema after ICH. At the same time, we also observed that in the $\mathrm{PI}(\mathrm{L})$ group compared with the ICH group, the relative expression of AQP4mRNA at each time point after surgery decreased, and brain water content decreased $(\mathrm{p}<0.05)$, suggesting that the appropriate dose of PAR1 inhibitor may be down-regulated. The expression level of AQP4mRNA further reduced cerebral edema after ICH. The possible mechanism for this phenomenon is as follows: a suitable dose of PAR1 inhibitor can keep the appropriate degree of PAR1 activated, and studies have shown that PAR1 can upregulate the expression of heat shock protein (HSP) (25). On the one hand, HSP can increase the level of glutathione in cells, enhance the resistance of cells to oxygen free radical damage after cerebral hemorrhage, stabilize cell membranes and ion pumps, reduce $\mathrm{Ca}^{2+}$ overload and thereby reduce the expression of AQP4mRNA, and reduce the degree of cerebral edema; and when high doses of PAR1 inhibitors reduce the HSP expression after excessive inhibition of PAR1 activity, which is not conducive to reducing brain tissue edema.

\section{Conclusions}

Up-regulation of AQP4mRNA expression after ICH increases cerebral water content; and a suitable dose of PAR1 inhibitor can reduce cerebral edema after ICH in experimental cerebral hemorrhage rats. The mechanism may be related to down-regulation of AQP4mRNA expression. This study provides new evidence on the treatment of cerebral hemorrhage by PAR1 inhibitor.

\section{References}

1. Christensen MC, Morris S, Vallejo-Torres L. Neurological Impairment Among Survivors of Intracerebral Hemorrhage: The FAST Trial. Neurocritical Care 2012; 16 (2): 224-231.

2. Chu H, Tang Y, Dong Q. Protection of Vascular Endothelial Growth Factor to Brain Edema Following Intracerebral Hemorrhage and Its Involved Mechanisms: Effect of Aquaporin-4. PLOS ONE 2013; 8 (6): e66051.

3. Staykov D, Wagner I, Volbers B et al. Natural course of perihemorrhagic edema after intracer ebral hemorrhage. Stroke 2011; 42 (9): 26252629.

4. Miyazaki K, Abe Y, Iwanari H et al. Establishment of monoclonal antibodies against the extracellular domain that block binding of NMOIgG to AQP4. J Neuroimmunol 2013; 260 (1-2): 107-116.

5. Yool AJ. Aquaporins: multiple roles in the central nervous system. Neuroscientist 2007; 13 (5): 470-485.

6. Papadopoulos MC. Aquaporin-4 facilitates reabsorption of excess fluid in vasogenic brain edema. FASEB J 2004; 18 (11): 1291-1293.

7. Hua Y, Keep RF, Hoff JT et al. Thrombin preconditioning attenuates brain edema induced by erythrocytes and iron. J Cerebral Blood Flow Metab 2003; 23 (12): 1448-1154.

8. Striggow F, Riek M, Breder $\mathbf{J}$ et al. The protease thrombin is an endogenous mediator of hippocampal neuroprotection against ischemia at low concentrations but causes degeneration at high concentrations. Proc Nat Acad Sci US Amer 2000; 97 (5): 2264-2269.

9. Bederson JB, Pitts LH, Tsuji M et al. Rat middle cerebral artery occlusion: evaluation of the model and development of a neurologic examination. Stroke 1986; 17 (3): 472-476.

10. Mei L, Chen J, Lu D et al. Global test statistics for treatment effect of stroke and traumatic brain injury in rats with administration of bone marrow stromal cells. J Neurosci Methods 2003; 128 (1-2): 183-190.

11. Karamalis M, Langer K, Demessinov A et al. PCV11. The Use of Minimally Invasive Surgery (Mis) and Intraoperative Imaging Modalities in the Treatment of Intracerebral Hemorrhage (Ich): A Systematic Review Of The Literature. Value in Health 2014; 17 (7): A474-A475.

12. Huang J, Lu WT, Sun TQ. Upregulation and lysosomal degradation of AQP4 in rat brains with bacterial meningitis. Neurosci Lett 2014; 566:156-161.

13. Tourdias T, Mori N, Dragonu I et al. Differential aquaporin 4 expression during edema build-up and resolution phases of brain inflammation. J Neuroinflamm 2011; 8 (1): 143.

14. Wu N, Zi M, Hui P, Wang N et al. Progression on Molecular Mechanisms of Cerebral Edema. Med Pharm J Chin PLA 2016; 28 (6): 14-18. 


\section{$600-604$}

15. Benga G. Birth of water channel proteins - the aquaporins. Cell Biol Int 2003; 27 (9): 701-709.

16. Badaut J, Lasbennes F, Magistretti PJ, Regli L. Aquaporins in brain: distribution, physiology, and pathophysiology. J Cereb Blood Flow Methab 2002; 22 (4): 367-378.

17. Hua Y, Keep R F, Gu Y et al. Thrombin and brain recovery after intracerebral hemorrhage. Stroke 2009; 40 (3 Suppl): 88-89.

18. Shi D, Cui GY. Study on the mechanism of thrombin in cerebral hemorrhage. Chinese J Pract Nerv Dis 2014; 17 (10): 138-140.

19. Kaufmann R, Part S, Zieger M et al. The two-receptor system PAR-1/ PAR-4 mediates alpha-thmmbin-induced [ca $\left.\left.{ }^{\left({ }^{+}\right.}\right)\right]$(i) mobilization in human astrocytoma cells. Cancer Res Clin Oncol 2000; 126: 91-94.

20. Lee KR, Colon GP, Berz AL et al. Edeme from intrracerebral hemrrhage:the role of thrombin. J Neurosurg 1996; 84 (1): 91- 96.

21. Teng WY, Tian L, Liu HL et al. Hirudin, Nimbrane and AQP after experimental cerebral hemorrhage in rats $4 \mathrm{mRNA}$ expression and the role of cerebral edema. China J Modern Med 2006; 9: 1290-1293.
22. Zhou QB, Jia Q, Zhang Y. Effect of baicalin on expression of thrombin receptor-1 and apoptosis in brain tissue of rats with intracerebral hemorrhage. China J Integrated Traditional Western Med 2010; 30 (12): 1302 -1305 .

23. Zheng GQ, Wang Y, Wang XT. Long-term dynamic expression of cerebral thrombin receptor-1 after cerebral hemorrhage in rats. China J Appl Physiol 2007; 23 (3): 328-332.

24. Wang KK. Calpain and caspase: can you tell me the difference? Trends Neurosci 2000; 23: 20-26.

25. Zhang J, Wang Y, Zhu P et al. siRNA-mediated silence of proteaseactivated receptor-1 minimizes ischemic injury of cerebral cortex through HSP70 and MAP2. J Neurol Sci 2012; 320 (1-2): 6.

26. Liu SJ, Zukin RS. Ca2+-permeable AMPA receptors in synaptic plasticity and neuronal death. Trends Neurosci 2007; 30 (3): 126-134.

Received December 18, 2020. Accepted April 6, 2020. 